\title{
Digital appendix 4. Authors associated with prominent syndication agencies and
}

agents

Mary Albert, Grant Allen, S. Baring-Gould, Frank Barrett, J. M. Barrie, William Black, Walter Besant, Guy Boothby, James S. Borlase, Hjalmar H. Boyesen, Frederick Boyle, Mary Elizabeth Braddon, Rhoda Broughton, Robert Buchanan, Frances Hodgson Burnett, Hall Caine, Sir Gilbert Campbell, Ina Leon Cassilis, Wilkie Collins, Hugh Conway (Frederick John Fargus), Marie Corelli, S. R. Crockett, Mary Angela Dickens, Edmund Downey, Arthur Conan Doyle, Harry Stillwell Edwards, Selwyn Eyre, B. L. Farjeon, George Manville Fenn, J. Monk Foster, Jessie Fothergill, R. E. Francillon, Alice French, Charles Gibbon, Ernest Glanville, H. Rider Haggard, Iza Duffus Hardy, Thomas Hardy, Joel Chandler Harris, Bret Harte, Joseph Hatton, Julian Hawthorne, G. A. Henty, Anthony Hope, E. W. Hornung, William Dean Howells, Margaret Wolfe Hungerford, Sarah Orne Jewett, Rudyard Kipling, William Le Queux, John K. Leys, Eliza Lynn Linton, Henry W. Lucy, Justin McCarthy, George MacDonald, Katherine S. MacQuoid, Arthur W. Marchmont, Florence Marryat, Helen Mathers, L. T. Meade, George Meredith, Mary Louisa Molesworth, J. Fitzgerald Molloy, J. E. Muddock, Rosa Mulholland, David Christie Murray, W. E. Norris, Margaret Oliphant, “Ouida” (Louise de la Ramee), David Pae, James Payn, Elizabeth Stuart Phelps, Eden Phillpotts, Arthur Quiller-Couch, Charles Reade, Compton Reade, Mayne Reid, "Rita” (Eliza Humphreys), F. W. Robinson, Dora Russell, W. Clark Russell, John Saunders, Adeline Sergeant, James Simson, Hawley Smart, Emily Spender, “John Winter Strange” (Henrietta Eliza Vaughan Stannard), Robert Louis Stevenson, Frank R. Stockton, Annie S. Swan, Frederick Talbot, Mary 
H. Tennyson, Lily Tinsley, Mark Twain, Jules Verne, H. G. Wells, William Westall, Stanley Weyman, Walter Wood, and Emile Zola.

Most of these authors are British, but some are American (Boyesen, French, Harris, Harte, Hawthorne, Hay, Jewett, Stillwell, and Twain), Canadian (Allen and Parker), French (Verne and Zola) and Australian (Boothby). 